\title{
Microscopic Measurement of Second Harmonic Generation from Chiral Surfaces
}

\author{
Daisuke Tokunaga,* Hideaki TaKeChI,* Jian-Hua YIn,* Hitoshi WataraI, ${ }^{* \dagger}$ and \\ Takahiro OHDE**
}

\author{
*Department of Chemistry, Graduate School of Science, Osaka University, \\ Machikaneyama-machi, Toyonaka, Osaka 560-0043, Japan \\ **CASI, Osaka University, 2-1 Yamadaoka, Suita, Osaka 565-0871, Japan
}

\begin{abstract}
A microscopic measurement system of circular dichroic spectra of second harmonic generation, which is applicable to detect the chirality of micro-region of molecular aggregates, has been investigated. The performance of the system was demonstrated by the discrimination of chirality of molecular aggregates of porphyrin on a glass plate.
\end{abstract}

(Received September 20, 2008; Accepted October 3, 2008; Published February 10, 2009)

\section{Introduction}

Second harmonic generation (SHG) spectroscopy has been applied to solid and liquid interfaces, because of its high interfacial selectivity and its sensitivity for the speciation and orientational characterization of surface or interfacial molecules including biological molecules. ${ }^{2}$ Especially, the application to the liquid/liquid interfaces has been developed with great interest, since there are so few measurement methods applicable to such soft interfaces..$^{3-6}$ Furthermore, since it was reported that SHG can be used for the detection of chirality of interfaces, ${ }^{7}$ the circular dichroic behavior of SHG has been studied mainly for solid and film surfaces. ${ }^{8-13}$ Chirality of the monolayer on air/water interface has been detected from the difference in SHG intensities for the left- and right-circular polarized light beams. ${ }^{14}$ Imaging of the chiral monolayer on a solid surface has attained detection of SHG-circular dichroism (CD) from the surface by an intensifier-coupled $\mathrm{CCD}$ camera. ${ }^{9}$ Recently, the first application of SHG-CD measurement for liquid/liquid interfaces was performed in our laboratory. ${ }^{15}$ Chirality of J-aggregate of diprotonated tetrakis(4-sulfonatephenyl)porphyrin with cetyltrimethylammonium cation formed at the heptane/water interface was measured by applying a totally internal reflection mode. It was shown that the interfacial molecular aggregate formed at the liquid/liquid interface could produce optically chiral SHG signals. In the present study, we have constructed a microscopic SHG-CD detection system to observe the chirality of micro-region of film or molecular aggregate, which is usually inhomogeneously formed on surfaces or interfaces. A microscopic method is necessarily required to examine chiral inhomogenity of interfacial aggregates and to acquire SHG-CD images of soft interfaces.

\section{Experimental}

Second harmonic generation-circular dichroism microscope spectropolarimeter

Figure 1 shows a schematic illustration of a newly constructed apparatus, where a Ti:sapphire laser (Maitai, Spectra-physics, USA; $80 \mathrm{MHz}, 100 \mathrm{fs}$, tuning range of $780-920 \mathrm{~nm}$ ), a beam expander (55577G, Edmund, USA), an ND filter (ND05B, ND10B and ND20B, THORLABS, USA), a polarizer (47327G, Edmund, USA), a half wave plate (AHWP05M-950, THORLABS, USA), a quarter wave plate (AQWP05M-950, THORLABS, USA), a dichroic mirror (order made, SURUGA SEIKI, Japan), a tube lens (Nikon, Japan), objective lens (LU Plan Fluor 100/0.90, Nikon, Japan), short-wavelength pass filters (BG38, BG39, CVI laser), fluorescence cut filters (V40, ASAHI GLASS, Japan), a photomultiplier (PMT) (Hamamatsu, Japan), mirrors (Optoquest, Japan), and lenses (Thorlabs, USA) were arranged on an optical bench. The intensity of the incident light from Ti:sapphire laser was controlled by the ND filter. A circular polarized light was formed by the polarizer, the half wave plate and the quarter wave plate. The incident light was focused on a sample by the objective lens. An SH light generated from the sample was collected by the same objective lens, then reflected by the dichroic mirror, and finally detected by the PMT. The remaining incident light and fluorescence

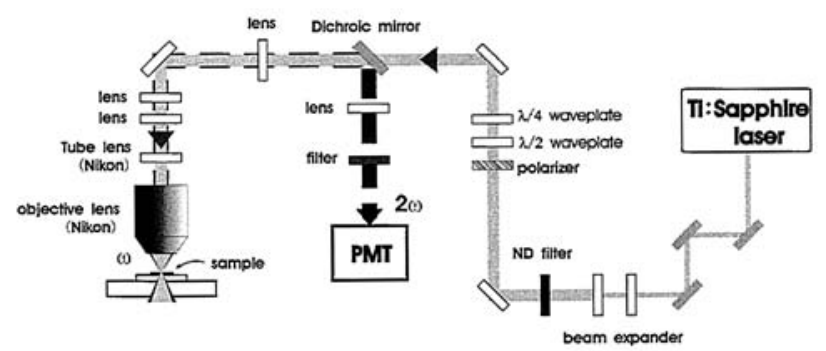

Fig. 1 Schematic illustration of the microscopic second harmonic

generation-circular dichroism spectropolarimeter.
† To whom correspondence should be addressed. E-mail: watarai@chem.sci.osaka-u.ac.jp 


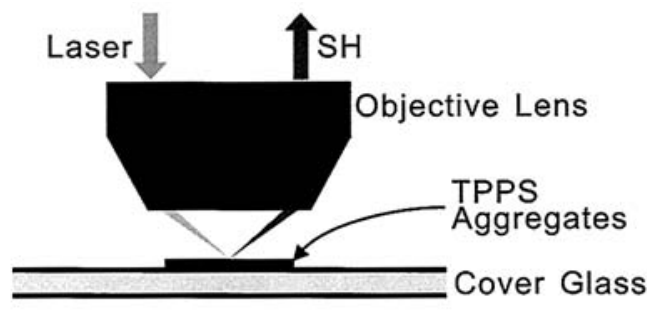

Fig. 2 Laser irradiation and SHG light collection method employed in the present research.

light were cut by short pass filters and fluorescence cut filters, respectively. Special caution was paid to arrange optical devices to minimize undesirable optical anisotropy. For the alignment of the SHG systems, a strong SHG light from a single granule crystal was used. When the measurement region was observed by the CCD camera, a mirror was introduced into a light path in a vertical microscope. In this study, measurement area was $c a$. $1 \times 1 \mu \mathrm{m}$. The wavelength scanning and data acquisition of SHG intensity were controlled by a personal computer.

As for the laser irradiation method, we employed a partial irradiation method, in which the incident beam was introduced through a shifted position from the center of the objective lens and the SHG light was collected by the same objective lens as shown in Fig. 2. It was found from our experiments that the partial irradiation method was better than a full irradiation method, in which an expanded irradiation light was transmitted through the whole area of the objective lens and the SHG light was collected by the same lens.

The observed SHG intensity was corrected by the laser power intensity and transmittance of a dichroic mirror, short pass filters and other mirrors, filters and lenses. The micro-SHG-CD intensity $\left(I_{\mathrm{SHG}-\mathrm{CD}}\right)$ was calculated by the equation:

$$
I_{\mathrm{SHG}-\mathrm{CD}}=\frac{I_{\mathrm{lcp}}-I_{\mathrm{rcp}}}{\frac{1}{2}\left(I_{\mathrm{lcp}}+I_{\mathrm{rcp}}\right)}
$$

where $I_{\mathrm{lcp}}$ and $I_{\text {rcp }}$ were SHG intensities of left-circular polarized light and right-circular polarized light, respectively.

$\mathrm{CD}$ spectra of solution and dispersion were measured by a conventional circular dichroism spectropolarimeter (J-820, Jasco, Japan). The CD spectra of a micro-region of the porphyrin aggregate on a glass plate was measured by holding the glass plate vertically with a microscope $\mathrm{CD}$ (abbreviated as micro-CD) measurement device, which is actually a pair of objective lenses installed in the sample compartment of the CD spectropolarimeter, which was recently constructed in our laboratory. ${ }^{16}$

\section{Materials}

Tetrakis(4-sulfonatophenyl)porphyrin (TPPS) was purchased from Frontier Scientific (USA). D- and L-tartaric acid, perchloric acid, hydrochloric acid and sodium chloride were purchased from Nacalai Tesque Inc. (Kyoto, Japan) and used as received. Sodium perchlorate (GR, Aldrich, USA) was used as received. Water was distilled and deionized by a Milli-Q system (Millipore, USA).

The J-aggregate of diprotonated TPPS ${ }^{4-}\left(\mathrm{H}_{2} \mathrm{TPPS}^{2-}\right)$ was prepared by mixing the aqueous solutions of $5.0 \times 10^{-5} \mathrm{M}$ TPPS $^{4-}, 3.0 \times 10^{-2} \mathrm{M}$ D- or L-tartaric acid, $0.10 \mathrm{M} \mathrm{HClO}_{4}$ or $\mathrm{HCl}$, and $0.50 \mathrm{M} \mathrm{NaClO}_{4}$ or $\mathrm{NaCl}$. The J-aggregate of $\mathrm{H}_{2} \mathrm{TPPS}^{2-}$ showed chirality well, following the chirality of the tartaric acids (Fig. 3). Keeping the solution for one day, the aggregate

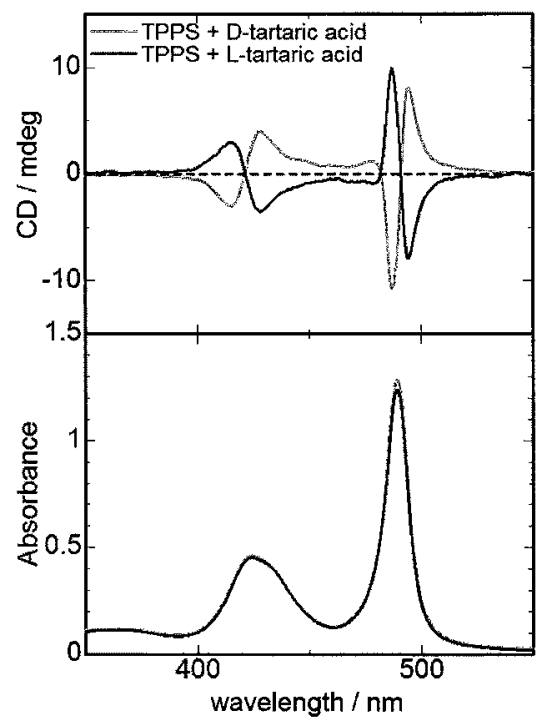

Fig. 3 Absorption and $\mathrm{CD}$ spectra of $\mathrm{H}_{2}$ TPPS $^{2-}$ aggregates in aqueous solution affected by D- or L-tartaric acid. These spectra were measured by a $1-\mathrm{mm}$ cell. $\left[\mathrm{TPPS}^{4}\right]=5.0 \times 10^{-5} \mathrm{M}$, [D-tartaric acid] $=3.0 \times 10^{-2} \mathrm{M},\left[\mathrm{HClO}_{4}\right]=0.10 \mathrm{M},\left[\mathrm{NaClO}_{4}\right]=0.50 \mathrm{M} ;\left[\mathrm{TPPS}^{4}\right]=$ $5.0 \times 10^{-5} \mathrm{M},[\mathrm{L}-$ tartaric acid $]=3.0 \times 10^{-2} \mathrm{M},\left[\mathrm{HClO}_{4}\right]=0.10 \mathrm{M}$, $\left[\mathrm{NaClO}_{4}\right]=0.50 \mathrm{M}$.

was concentrated by a centrifugal separation and transferred on a cover glass to be dried. The film was used as samples for SHG measurements.

\section{Results and Discussion}

The dispersion of J-aggregate of $\mathrm{H}_{2} \mathrm{TPPS}^{2-}$ showed specific absorption bands at 420 and $490 \mathrm{~nm}$, corresponding to the transition moments parallel to the short axis and the long axis of the J-aggregate, respectively (Fig. 3). The Cotton effect in the $\mathrm{CD}$ spectra of the J-aggregate in Fig. 3 reflected correctly the chirality of D- and L-tartaric acid at the two bands. The close distance interaction between porphyrin ring and chiral tartaric acid in the aggregate was thought to generate such enhanced optical chirality through the supra-molecular structure. ${ }^{17}$

The micro-SHG-CD spectra were measured in the wavelength range from 390 to $460 \mathrm{~nm}$. The observed micro-SHG-CD spectra of $\mathrm{H}_{2}$ TPPS $^{2-}$ aggregates on a glass plate, shown in Figs. 4(a) and 4(b), were affected by the chirality of D- and L-tartaric acids respectively, where the micro-CD spectra of the aggregate were shown together for comparison. The micro-SHG intensity depended on a measurement position, suggesting the microscopic-inhomogenity of the concentration and orientation of the aggregate. It was found that both spectra of micro-SHG$\mathrm{CD}$ and micro-CD showed similar apparent characteristics, though the shapes were not identical. The shapes of SHG and SHG-CD spectra were almost the same for the same sample. SHG spectra had a peak at $420 \mathrm{~nm}$ that was corresponding to the absorption peak of the short axis of $\mathrm{H}_{2} \mathrm{TPPS}^{2-}$ aggregate. In both chiral samples, although the SHG intensity was different for left-circular polarized light and right-circular polarized light, the shape of the SHG-CD spectra looked like a negative CD signal. It was simply expected that SHG-CD signal of $\mathrm{H}_{2} \mathrm{TPPS}^{2-}$ aggregates formed with D-tartaric acid gave a negative $\mathrm{CD}$ sign and that formed with L-tartaric acid had a positive $\mathrm{CD}$ sign. The results of our SHG-CD measurements were not in agreement 
(a) D-tartaric acid
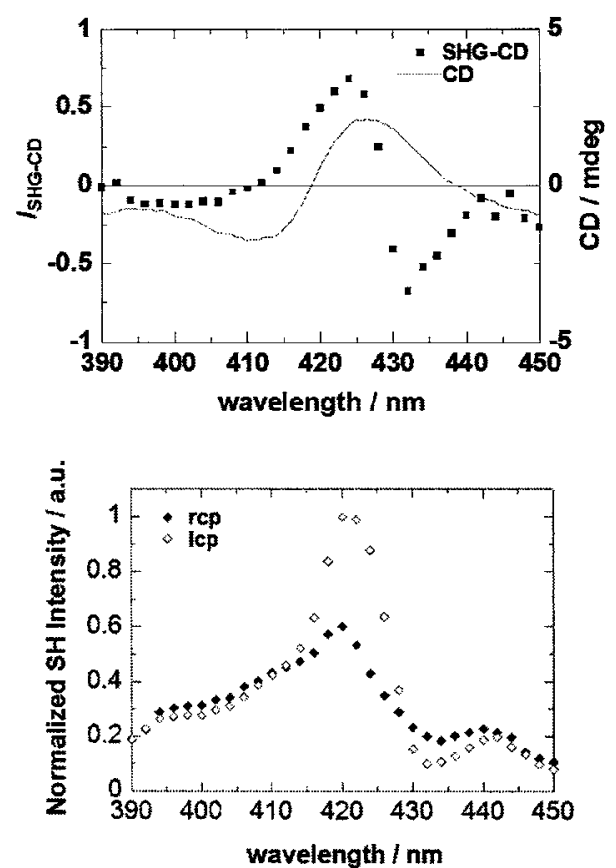

(b) L-tartaric acid
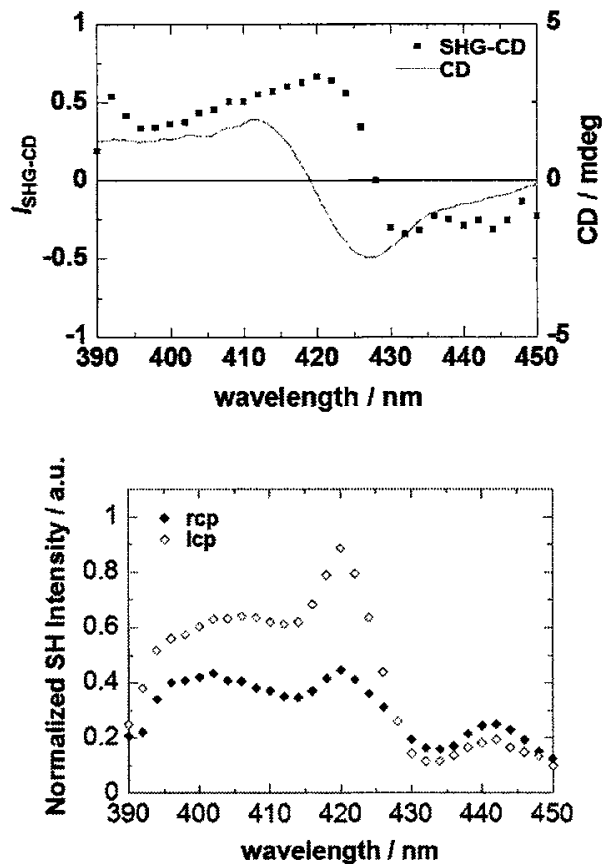

Fig. 4 Micro-SHG-CD and micro-CD spectra of $\mathrm{H}_{2}$ TPPS $^{2-}$ aggregate affected by D-tartaric acid (a) and L-tartaric acid (b). Micro-SHG: (a) [TPPS $\left.{ }^{4}\right]=1 \times 10^{-4} \mathrm{M}$, [D-tartaric acid] $=3 \times 10^{-2} \mathrm{M},[\mathrm{HCl}]$ $=0.10 \mathrm{M},[\mathrm{NaCl}]=0.50 \mathrm{M},\left(\right.$ b) $\left[\right.$ TPPS $\left.^{4}\right]=1 \times 10^{-4} \mathrm{M},[$ L-tartaric acid $]=3 \times 10^{-2} \mathrm{M},[\mathrm{HCl}]=0.10$ $\mathrm{M},[\mathrm{NaCl}]=0.50 \mathrm{M}$. Micro-CD: (a) $\left[\mathrm{TPPS}^{4}\right]=1 \times 10^{-4} \mathrm{M},[$ D-tartaric acid $]=5 \times 10^{-2} \mathrm{M},[\mathrm{HCl}]=$ $0.01 \mathrm{M},[\mathrm{NaCl}]=0.09 \mathrm{M},\left(\right.$ b) $\left[\mathrm{TPPS}^{4}\right]=1 \times 10^{-4} \mathrm{M},[$ L-tartaric acid $]=5 \times 10^{-2} \mathrm{M},[\mathrm{HCl}]=0.01 \mathrm{M}$, $[\mathrm{NaCl}]=0.09 \mathrm{M}$.

with this expectation, but it was found that the shape of SHG-CD spectra was clearly affected by the chirality of the tartaric acid.

The microscopic instrument constructed in the present study could detect SHG light, which was confirmed by the observation of a quadratic relation between the SHG intensity and the power of the incident laser beam. However, it was difficult to eliminate completely the anisotropic effect of optical parts. It was difficult to keep the quality of the circular polarized incident light after passing through several lenses and mirrors, and the circular polarized light became slightly elliptically polarized light at a sample position. The measurement of the intensity ratio at 0 and 90 degrees of the circular polarized light showed that the circular polarized light was distorted less than $15 \%$ at the sample position depending on the wavelength. Furthermore, the TPPS aggregates dried on a glass plate was accompanied by salt crystals, probably those of $\mathrm{NaCl}$ and tartaric acid. Such crystals might induce some optical artifacts due to their optical anisotropies, although a crystal-like position gave no significant SHG signals. In the present study, we measured film-like position to prevent the effect of any artifacts. In addition, we tried to measure the micro-SHG-CD spectra of the aggregates of bilirubin and bilirubin-BSA complex formed at the heptane/water interface. In the system of bilirubin-BSA complex, the SHG spectra were different from those of rasemic bilirubin, suggesting the preference of $M$-conformation in the bilirubinBSA complex, ${ }^{18}$ although it was seriously affected by the anisotropic optical property of the solid aggregate.

\section{Conclusions}

In the present study, we have constructed a microscopic second harmonic generation-circular dichroism spectropolarimeter system and tried to detect the optical chirality of micro-regions of the surface of chiral $\mathrm{H}_{2}$ TPPS $^{2-}$ aggregates. The system could measure SHG signal from $c a .1 \times 1 \mu \mathrm{m}$ micro-region of dried $\mathrm{H}_{2}$ TPPS $^{2-}$ aggregates on a glass plate. However, it was pointed out that the micro-SHG-CD spectra were seriously distorted by the anisotropy of the optical devices in the system. In order to avoid such non-ideality, it will be required to simplify the optical system and to improve the matching of the optical devices to maintain the ideal circular polarizability of incident beam. Also, the optical anisotropy of the aggregate should be considered to get correct SHG-CD spectra. The present knowledge will be highly useful for the construction of a second harmonic generation-circular dichroism imaging microscopy, which is applicable to soft interfaces such as liquid-liquid interface and bio-cell membrane.

\section{Acknowledgements}

This study was supported by a Grant-in-Aid for Scientific Research (S) (No. 16105002) and in part by "Special Coordination Funds for Promoting Science and Technology: Yuragi Project" of the Ministry of Education, Culture, Sports, Science and Technology, Japan.

\section{References}

1. R. M. Corn and D. A. Higgins, Chem. Rev., 1994, 94, 107.

2. C. Odin, Y. L. Grand, A. Renault, L. Gailhouste, and G. Baffet, J. Microscopy, 2008, 229, 32. 
3. K. Fujiwara, H. Monjushiro, and H. Watarai, Rev. Sci. Instrum., 2005, 76, 023111.

4. D. Pant, M. L. Guennec, B. Illien, and H. H. Girault, Phys. Chem. Chem. Phys., 2004, 6, 3140.

5. C. L. Beildeck, W. H. Steel, and R. A. Walker, Faraday Discuss., 2005, 129, 69.

6. N. Nishi, R. Ishimatsu, M. Yamamoto, and T. Kakiuchi, $J$. Phys. Chem. C, 2007, 111, 12461.

7. J. M. Hicks, T. Petralli-Mallow, and J. D. Byers, Faraday Discuss., 1994, 99, 341.

8. A. M. Pena, T. Boulesteix, T. Dartigalongue, and M. C. Schanne-Klein, J. Am. Chem. Soc., 2005, 127, 10314.

9. M. A. Kriech and J. C. Conboy, J. Am. Chem. Soc., 2005, 127, 2834.

10. V. Rodriguez, G. Koeckelberghs, and T. Verbiest, Chem. Phys. Lett., 2007, 450, 76.

11. J. Kawamata, H. Yamaki, R. Ohshige, R. Seike, S. Tania, Y.
Ogata, and A. Yamagishi, Colloids Surf., A, 2008, 321, 65.

12. X. Li, J. Willits, S. T. Cundiff, I. M. P. Aarts, A. A. E. Stevens, and D. S. Dessau, Appl. Phys. Lett., 2006, 89, 022102 .

13. F. Araoka, N. Y. Ha, Y. Kinoshita, B. Park, J. W. Wu, and H. Takezoe, Phys. Rev. Lett., 2005, 94, 137801.

14. T. Manaka, R. Tamura, and M. Iwamoto, Colloids Surf., A, 2006, 284, 409.

15. K. Fujiwara, H. Monjushiro, and H. Watarai, Chem. Phys. Lett., 2004, 394, 349.

16. A. Matsugaki, H. Takechi, H. Monjushiro, and H. Watarai, Anal. Sci., 2008, 24, 297.

17. O. Ohno, Y. Kaizu, and H. Kobayashi, J. Chem. Phys., 1993, 99, 4128.

18. J.-H. Yin and H. Watarai, Anal. Bioanal. Chem., 2007, 389, 895. 